\title{
Detachment of the Amplatzer Device after Interventional Closure of a Mitral Prosthesis Paravalvular Leak
}

\author{
Alexandra Pavel. ${ }^{1}$
}

\begin{abstract}
Background: Paraprosthetic leak is a complication of mitral and aortic valve implantation interventions, that can be treated either through a surgical procedure (repair or replacement of the prosthesis) or by means of a less-invasive percutaneous transcatheter approach. Nevertheless, this percutaneous intervention carries complications on its own, one of them being displacement of the occluder device. Results: This is the case of a 66-year-old patient, with a clinical history of two mitral valve replacement procedures, who presented with dyspnea, fatigue, and peripheral edema. Echocardiographic findings described severe mitral regurgitation due to the presence of a paravalvular leak. Considering the high perioperative mortality, renal and respiratory impairment, and the fact that this reoperation would have been the third cardiac surgery for the patient, percutaneous transcatheter closure of the leak was recommended. Four days after the successful procedure, a checkup echocardiogram revealed the migration of the occluder device to the left atrial cavity. Even though the operative risk was high and the prognosis poor, the only available treatment option was the surgical management that achieved the removal of the device and replacement of the diseased prosthesis. Conclusion: In conclusion, albeit a less invasive approach, with lower risks and better outcomes, the percutaneous intervention for leak closure has some clinically relevant complications that must be addressed promptly. One of these complications - detachment of the occluder device - even if quite rare, is a significant event that requires immediate surgical or interventional approach. A careful postoperative checkup is therefore essential to detect any complication and to address it directly.
\end{abstract}

Key Words: Heart Valve Prosthesis Implantation; Prosthesis Failure; Endoleak; Septal Occluder Device; Reoperation (Source: MeSH-NLM).

\section{Introduction}

Paravalvular leakage is a relatively rare complication of mitral or aortic replacement procedures and refers to an abnormal passageway between two cardiovascular chambers, which results in the backflow of blood through the space situated between the prosthesis and the valvular annulus.' It occurs more frequently after mechanical prosthesis implantation and is more commonly observed in the setting of mitral than aortic valve replacement. Paraprosthetic leaks are often caused by infective endocarditis, fibrosis or calcification of the annulus, that increase the risk of suture dehiscence, or by surgical technical problems leading to inappropriate sealing of the prosthesis to the native annulus. ${ }^{2}$ Treatment options for paravalvular leaks are openheart surgery and the less invasive percutaneous transcatheter closure of the defect. However, reported interventional complications are: access site bleeding, cardiac tamponade, arrhythmias, embolization of the device and prosthetic valve obstruction.3,4

\section{The Case}

A 66-year-old man presented with symptoms of congestive heart failure - dyspnea and fatigue during mild activity (New York Heart Association [NYHA] functional class III), swollen legs and ankles, ascites - and a mitral paravalvular leak on the echocardiogram. The patient's clinical history included a previous presentation (2004) for rheumatic mitral valvulopathy, for which he had undergone a mitral mechanical prosthesis implantation, and a reoperation for subacute mitral endocarditis nine years later, when the diseased prosthesis was replaced by another mechanical heart valve; in 2004, simultaneously with the mitral valve replacement, a coronary artery bypass graft surgery was performed, anastomosing left internal mammary artery (LIMA) to the left anterior descending artery (LAD).
At the current presentation (May 2017), transthoracic echocardiography showed mitral prosthetic detachment, with a posterolateral paraprosthetic leak that caused severe mitral regurgitation. Further transthoracic echocardiographic evaluation revealed a severely dilated left atrium, dilated right cavities, mild degenerative aortic stenosis and insufficiency, moderate tricuspid regurgitation and secondary pulmonary artery hypertension; the global ejection fraction was $40 \%$. The patient also had a history of hypertension, atrial fibrillation, and dyslipidemia. Laboratory tests showed a mild anemia with $10.7 \mathrm{~g}$ hemoglobin/dL, serum creatinine $1.7 \mathrm{mg} / \mathrm{dL}$ and minimal biologic alterations consistent with the cardiac failure (serum urea $78 \mathrm{mg} / \mathrm{dL}$, AST $92 \mathrm{U} / \mathrm{L}$ ). The patient also suffered from restrictive lung disease with impaired respiratory function. Left-heart coronary angiography showed a functional LIMA to LAD anastomosis and minimal coronary artery disease, except for the left anterior descending artery which was completely occluded proximally.

Given the patient's NYHA class III heart failure and severe mitral regurgitation, but also the capacity of medical therapy of only improving the symptoms without treating the cause, closure of the defect was strongly indicated. 5 The available methods of repairing the defect were either cardiac surgery or percutaneous intervention. Knowing that repeated cardiac surgery is associated with high morbidity and mortality, that a surgical reintervention for prosthesis replacement would be less effective given the presence of valvular endocarditis, but also due to notable advantages of an interventional procedure (shorter hospital stays, early resumption of daily activities, lower risk of complications), the percutaneous leak closure seemed more appropriate. 5 Consequently, the surgical consult concluded that the patient's multiple co-morbidities (chronic kidney disease: eGFR [estimated glomerular filtration rate] $=43 \mathrm{~mL} / \mathrm{min} / 1.73 \mathrm{~m}^{2}$, restrictive lung disease $[\mathrm{FEV} / \mathrm{FVC}$ ratio $=81 \%$ of predicted value] $)$ and high operative mortality (EuroSCORE II $=11.47 \%$ ) contraindicated the surgical

1 "Carol Davila" University of Medicine and Pharmacy, Bucharest, Romania.

About the Author: Dr. Alexandra Pavel is affiliated with the "Carol Davila" University of Medicine and Pharmacy, Bucharest, Romania. 
approach, hence prompting to proceed with the percutaneous transcatheter closure.

The intervention was performed with the patient under general anesthesia and with the help of 3 -dimensional transesophageal echocardiography monitoring. The selected device for the intervention was The Amplatzer ${ }^{\circledR}$ Vascular Plug III due to the crescentic shape of the paravalvular leak.3 After the successful deployment of the device at the site, transesophageal echocardiography demonstrated mild lateral paraprosthetic leak and a functional mechanical prosthesis. The patient's recovery was uneventful, except for the fourth postprocedural day, when checkup transthoracic echocardiography revealed a free 10- $\mathrm{mm}$ hyperechoic structure floating in the left atrium, consistent with the Amplatzer device dislodged from the deployment place (Figure 1).

Figure 1. Transesophageal Echocardiography Revealing the Amplatzer Vascular Plug III Device Floating in the Left Atrium.

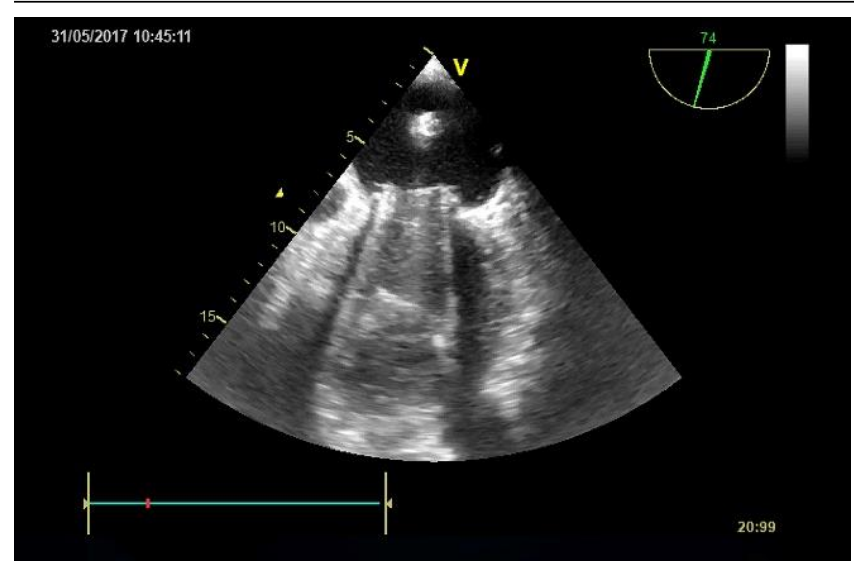

To solve this problem, although the perioperative mortality was high, and the surgical prognosis was poor, the patient's third cardiac surgery was performed. The Amplatzer Vascular Plug III was removed under direct vision (Figure 2). A mitral valve replacement was achieved simultaneously using a bioprosthesis; furthermore, the tricuspid valve was repaired through an annuloplasty and an aortic valvuloplasty was performed (shaving cusps' free margins, subcommissural annuloplasties). Therefore, the surgical approach was the best option in the present case, for the following reasons: firstly, the mitral regurgitation could only be solved through a surgical procedure (even if the dislodged device would have been removed in an interventional manner), and secondly, the surgical procedure allowed additional repair of aortic and tricuspid valves. The patient recovered slowly after the reoperation and was discharged 20 days later, hemodynamically and respiratory stable. Seven-month follow-up transthoracic echocardiography showed functional mitral bioprosthesis, with no paraprosthetic leaks, mild biatrial dilation and improved ventricular ejection fraction. No heart failure symptoms were present, and the patient had resumed daily physical activity.

\section{Discussion}

Paravalvular leaks are a relatively rare complication of valve replacement procedures, that can be addressed either through surgery or a percutaneous approach. Devices used to achieve an interventional procedure are not specifically designed for paravalvular leak closure, thus the reluctance of applying this method of treatment; however, with respect to the shape and size of the defect, assessed by echocardiography, there are several septal occluder devices available for closure of the paraprosthetic leak. ${ }^{6}$ Interventional closure of a paraprosthetic leakage holds several clinically relevant complications: cardiac tamponade can be related to transseptal catheterization or guidewire left ventricular perforation; atrial fibrillation and complete heart block are rare complications that can resolve under medical treatment or pacemaker implantation; prosthetic valve obstruction can almost always be recognized immediately by echocardiography.5, 7

Embolization of the device is another post-procedural complication that requires emergent reintervention or cardiac surgery, depending on patient's history, clinical and echocardiographic findings, comorbidities and mortality. Although no large prevalence studies approached this complication, three other cases of device displacement in the setting of mitral paraprosthetic leak closure were reported, where correction was achieved through surgical approach.4, 8, 9 Another case of mitral paravalvular leakage that was closed with an Amplatzer Plug III device was followed by a late occluder dislodgement; this matter was addressed through another successful interventional procedure. ${ }^{10}$

Figure 2. Amplatzer Vascular Plug III Device after Surgical Removal from the Left Atrial Cavity.

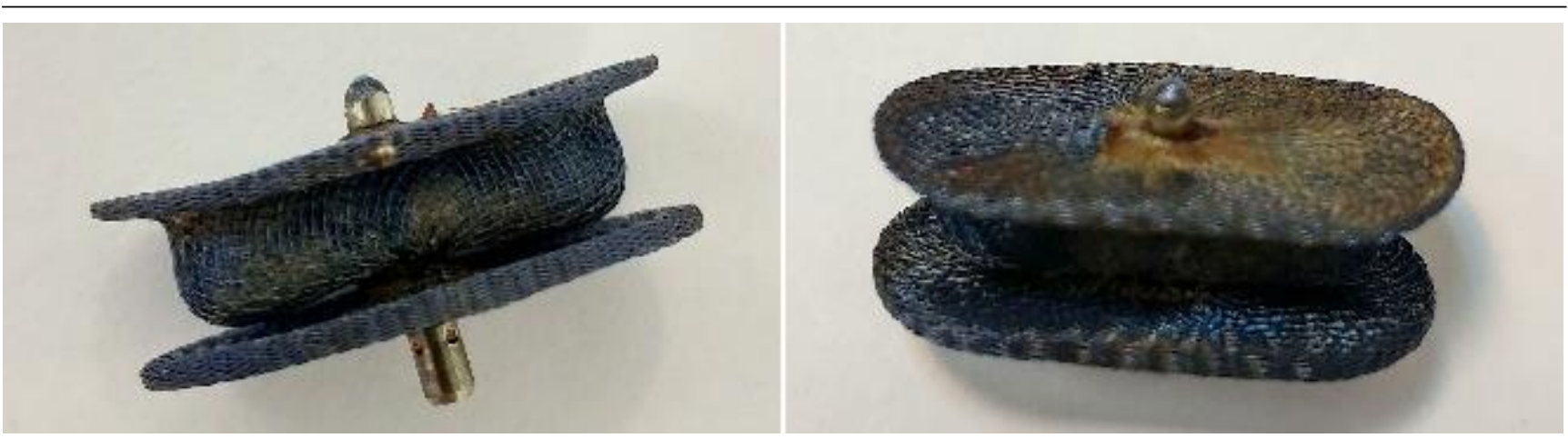

Because of high operative risk, important comorbidities, and given the considerable advantages of the interventional procedure, and suitability for patients with previous cardiac surgical interventions, percutaneous closure of the paravalvular leak was the method of choice in the present case. However, the early detachment of the occluder device had to be addressed through a surgical procedure that allowed not only the repair of the mitral regurgitation but also that of the aortic and tricuspid valves failure.

Although percutaneous closure of a paravalvular leak holds a lower mortality risk than the surgical approach, there are significant post- procedural complications, such as displacement of the occluder device, leading to severe regurgitation, that requires repeated intervention. Therefore, thorough echocardiographic follow-up in patients who had undergone cardiac valve surgery or percutaneous intervention is of paramount importance to ensure an early detection of a possible complication and come up with the most appropriate solution. In addition, one must keep in mind that no intervention is perfect, thus concentrating on choosing the best treatment, according to the patient's specifications, and being prepared for any unwanted effect. 


\section{References}

1. Sivakumar K. Transcatheter closure of paravalvular leaks - how do I do it? Indian Heart J. 2013 May; 65(3): 289-294.

2. Smolka C, Wojakowski W. Paravalvular leak - important complication after implantation of prosthetic valve. E-Journal of Cardiology Practice. $2010 \mathrm{Nov} ; 9(8)$

3. Branny M, Januška J, Škňouřil L, Holek B, Dorda M, Gajdǔšek L. Management of paravalvular leaks. Cor et Vasa. 2012 May-Jun;54(3):e 151-e155.

4. Yuan SM, Shinfeld A, Raanani E. Displacement of the Amplatzer occluder device from the mitral paraprosthetic leak. Interact Cardiovasc Thorac Surg. 2008 $\operatorname{Dec} ; 7(6): 1131-1133$.

5. Eleid MF, Cabalka AK, Malouf JF, Sanon S, Hagler DJ, Rihal CS. Techniques and Outcomes for the Treatment of Paravalvular Leak. Circ Cardiovasc Interv. 2015 Aug;8(8):e001945.
6. Gafoor S, Franke J, Bertog S, Lam S, Vaskelyte L, Hofmann I, et al. A Quick Guide To Paravalvular Leak Closure. Interv Cardiol. 2015 May;10(2):112-117.

7. Eeckhout E, Carlier S, Lerman A, Kern M. Handbook of Complications durin Percutaneous Cardiovascular Interventions. United Kingdom: Informa Healthcare; Informa UK Ltd; 2007. 270-272 p.

8. Ussia GP, Scandura S, Calafiore AM, Mangiafico S, Meduri R, Calassi AR, et al. Images in cardiovascular medicine. Late device dislodgement after percutaneous closure of mitral prosthesis paravalvular leak with Amplatzer muscular ventricula septal defect occluder. Circulation. 2007 Feb 27;115(8):e208-10.

9. Codinho AR, Almeida PB, Sousa C, Concalves A, Silva JC, Maciel MJ. Late device embolization in a persistent mitral paravalvular leak. Rev Port Cardiol. 2015 Apr;34(4):291.e 1-4.

10. Arzamendi D, Li CH, Serra A. Late embolization of a vascular plug III device after mitral paravalvular leak closure. Catheter Cardiovasc Interv. 2013 Nov 15:82(6):E831-4.

\section{Acknowledgments}

None.

\section{Conflict of Interest Statement at Funding}

The Authors have no funding, financial relationships or conflicts of interest to disclose.

Author Contributions

Conception and design the work/idea, Collect data/obtaining results, Analysis and interpretation of data, Write the manuscript, Critical revision of the manuscript, Approval of the final version: AP.

Cite as:

Pavel A. Detachment of the Amplatzer Device after Interventional Closure of a Mitral Prosthesis Paravalvular Leak. Int J Med Students. 2018 Jan-Apr;6(1):25-27. 